\title{
Cayetano Heredia: el punto de partida y una dilatada enfermedad
}

\author{
Cayetano Heredia: A departure and a prolonged illness
}

\author{
E. Eduardo Zárate Cárdenas
}

'Profesor Principal, Departamento de Salud Pública y Medicina Preventiva, Facultad de Medicina, Universidad Nacional Mayor de San

Marcos, Lima, Perú

\begin{abstract}
Resumen
Sobre el profesor Cayetano Heredia se ha construido una imagen laudatoria de su labor como primer decano de la Facultad de Medicina. Sin embargo, esta es una situación de espacios interrumpidos por las crisis políticas y económicas de las primeras décadas de la república. De igual manera, poco se ha tratado sobre su salud. La revisión de fuentes primarias nos permite valorar más aún su fecunda tarea, en la medida que esta se da en medio de una enfermedad crónica que lo llevaría a la muerte. Asimismo, se discute las causales sobre su no continuidad en el cargo de decano a pesar de una labor reconocida por la sociedad médica.

Palabras clave: Cayetano Heredia, educación médica.

Abstract

Much praise has been given to Cayetano Heredia's role as first dean of the Medical College of Lima. Less common are mentions on interruptions this work suffered as a result of the Peru's first decades' economic turmoil and Heredia's own ailing health. This text draws on primary sources to share a new look on Heredia's remarkable achievements, the reasons behind his departure from his role -despite ample recognition of his work in Peruvian medical society- and the chronic illness that would lead to his ultimate death.
\end{abstract}

Keywords: Medical College of Lima, Cayetano Heredia, medical education,

An Fac med. 2013;74(3):249-53

\section{INTRODUCCIÓN}

Los escritos clásicos sobre la vida del profesor Cayetano Heredia han recibido atención, pero quizás no la suficiente, sobre su accionar como primer Decano de la Facultad de Medicina del Perú y sus últimos días. Los historiadores médicos dejaron de lado las condiciones de su salud física con las que convivió y desarrolló su fecundo trabajo.

Acompañando a su labor de organizador de la Escuela de Medicina (EM), en compleja relación de causa y efecto se registra un deterioro en su salud física, con ritmos variables por cierto. Este es un aspecto poco tratado entre sus clásicos biógrafos ${ }^{(1-3)}$. Nuestra investigación encontró manuscritos que descubren una enfermedad crónica que lo llevará a la muerte, aspecto que demostramos en el presente artículo.

Los estudios de la historia de la medicina peruana habían evitado hablar sobre las vicisitudes en la formación de la EM, probablemente para encontrar una continuidad ininterrumpida entre la medicina de la colonia y la de la república. Sin embargo, serán las discontinuadas las que formarán parte de la constitución de la EM debidas a la inestabilidad política y fiscal de las primeras décadas de la república. Investigaciones sobre las nuevas fuentes nos permiten comprender que para el auge y posicionamiento de la EM tiene que considerarse, entre otros factores, los cambios

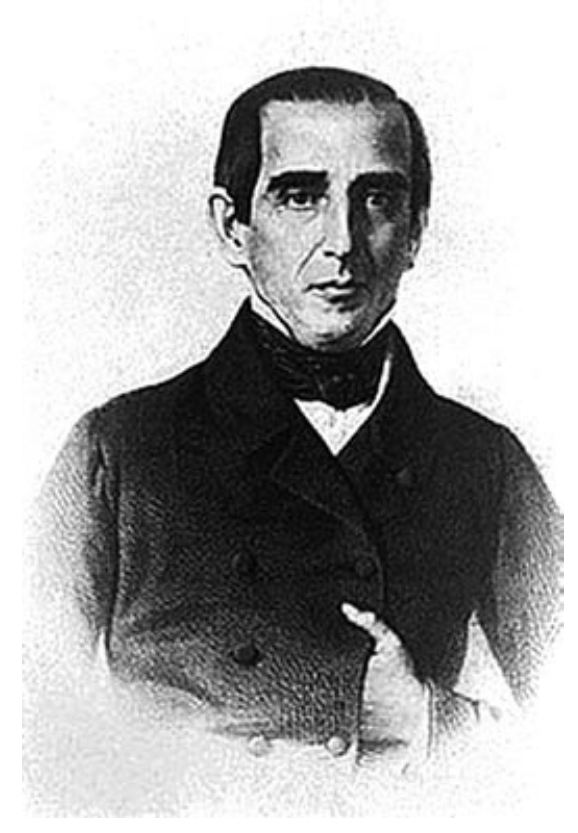


de la primera modernización del país en el marco de la bonanza económica dada por el guano de las islas y la relativa paz social de los gobiernos de Ramón Castilla ${ }^{(4)}$. Así, un conjunto articulado, no planificado, de hechos sociales y la mejora económica del país contribuyeron al posicionamiento científico y político de los egresados de la EM. En este escenario, la figura de Cayetano Heredia tuvo una eficaz capacidad organizativa, un adecuado juicio para identificar personajes científicos con futuro y encarnó los ideales sobre lo que debería ser la Medicina en el Perú.

El trabajo académico de Cayetano Heredia se dio principalmente entre 1831 y 1860 , y es a la vez el tránsito de la profesión médica de segundo orden hacia posiciones de conducción en las políticas sanitarias. A este proceso contribuyeron las opiniones científicas sobre las propiedades del guano de las islas, la fecunda capacidad organizativa de Cayetano Heredia. Esta labor le permitió formar discípulos que alcanzaron relieve en la historia médica peruana, como José Casimiro Ulloa, Camilo Segura, Francisco Rosas; ellos divulgaron ideas sobre ciencias e higiene en publicaciones nacionales, y fundaron la $\mathrm{Ga}$ ceta Médica de Lima, en 1856, la cual alcanzó difusión en Lima, Arequipa, Cusco, Guayaquil y Santiago de Chile.

\section{LOS INICIOS}

Su principal biógrafo C. Paz Soldán (1) anota que nació en Catacaos el 5 de agosto de 1797, hijo de Manuela Sánchez y Pablo Heredia. La primera década de su existencia es desconocida. De igual manera, se desconoce cómo llegó a Lima y se incorpora al convento de San Francisco siendo adolescente (14 años). Por estos años ya estaba formándose el Real Colegio de Medicina y Cirugía (CRMC). Se prepara en el Colegio de Príncipes para fortalecer sus conocimientos en matemáticas y latín. A los 15 años ingresaría al CRMC, siendo protegido del rector del colegio. $\mathrm{Al}$ terminar sus estudios ingresa como disector de Anatomía y parece mantu- vo vínculos con Hipólito Unanue, por cuanto J. Lastres ${ }^{(5)}$ ha publicado una carta en la cual $\mathrm{H}$. Unanue encargó a Cayetano Heredia la autopsia de Francisco de Paula Vigil, muerto repentinamente (1826).

Las circunstancias que llevaron al nombramiento de Cayetano Heredia como rector del colegio no son claras; tampoco los motivos de recibir un colegio en plena crisis (cierres temporales y crisis fiscal del país). Paz Soldán (1) afirma que las razones son vínculos políticos y amicales con el presidente de turno, el general Orbegoso. El inicio de una trayectoria en la conducción de la Escuela de Medicina (EM) se dio el 18 de marzo de 1834. Este primer período directivo será corto, porque a los diez días de ser nombrado solicitó permiso y pidió que se nombre un rector interino por tener que ausentarse, “... debiendo marchar mañana a prestar mis servicios en clase de cirujano mayor del ejército..." (6). El pedido fue aceptado y se nombró a Francisco Fuentes como rector interino.

La prioridad por cumplir misiones militares y las facilidades de concederle permisos por parte del gobierno hablan de vínculos fluidos entre Cayetano $\mathrm{He}$ redia y el poder militar de esa época. El camino de Heredia en la organización médica militar no está claro, pero Paz Soldán ${ }^{(1)}$ anota que ascendió rápidamente en mérito a ser miembro de la campaña del General Orbegoso contra las fuerzas de Agustín Gamarra, llegando a la clase de Cirujano en Jefe. Los vínculos de Cayetano Heredia y el poder militar le permitieron que a los 39 años ocupara el alto cargo de Inspector General de Hospitales. La labor de Cayetano Heredia en el espacio militar es reconocida a través de una condecoración. El 18 de abril de 1837 se nombró a Cayetano Heredia miembro de la Legión de Honor Nacional ${ }^{(7)}$. Esta legión era parte de la meritocracia pública y limitada a un número de empleados civiles. En diciembre de 1836 había sido nombrado miembro de la Legión de Honor el Protomédico General del Estado y con la incorporación de Cayetano
Heredia fueron solo los únicos médicos pertenecientes a esta institución ${ }^{(a)}$.

Desde inicios de 1838 hasta el primer semestre de 1839 es probable que el Colegio continuara cerrado. Los manuscritos del Archivo General de la Nación y de los Archivos de la Facultad de Medicina de Lima no registran información que muestren una vida académica. Los documentos encontrados corresponden, en general, a oficios de la prefectura que citaba a actuaciones de homenaje a personajes del gobierno. Puede concluirse que este primer período dedicado a la marcha de la EM, por parte de Cayetano Heredia, fue corto, por cuanto tuvo que compartirlo con quehaceres militares y fue escasa su contribución en la organización de los estudios de Medicina.

\section{CREACIÓN Y ENFERMEDAD}

Sobre el periodo anterior existen controversias acerca de las razones que motivaron la interrupción de su cargo. Así, Lastres ${ }^{(6)}$ refiere que durante tres años se aleja Cayetano Heredia de la dirección del Colegio "... Hay un interregno en la actividad creadora de $\mathrm{CH}$, contrariedades políticas lo alejan del Colegio durante tres años (1839-1842)". Nuestra investigación encontró manuscritos inéditos del Archivo General de la Nación, donde Cayetano Heredia renuncia por razones de salud el siete de agosto de 1840 .

“... a tiempo que adolezco de un padecimiento penoso de pecho, ...estos últimos meses ha tomado un incremento tal,

\footnotetext{
${ }^{a}$ La Legión de Honor Nacional fue una institución destinada a recompensar los méritos de funcionarios civiles y militares abarcando todas las jerarquías. Todos sus miembros gozaban de una pensión de 60 pesos anuales. Fue creada por el Gral. Santa Cruz, en diciembre de 1835, e instalada en noviembre de 1836.

${ }^{\mathrm{b}}$ El Protomedicato fue eliminado en 1848 por decreto del gobierno de Ramón Castilla y sus funciones fueron trasladadas al Colegio de Medicina.
} 
que me priva de la esperanza de poder restablecerme completamente, por la experiencia estoy convencido que sólo a meses de separarme de todo trabajo activo intelectual y de toda ocupación, ...lograré reponerme en parte mi salud extremada y por este motivo es que hoy me veo en la obligación de dimitir el cargo de rector del Colegio... tengo sin duda Sr. ministro, muchos títulos para amar con ternura, esta casa a la que me miro como madre, donde comencé mi educación desde mis más tiernos años, a la que debo mi carrera" ${ }^{(8)}$.

Cayetano Heredia afirma que desde los últimos meses padece de una enfermedad crónica y añade que en los últimos meses se ha incrementado. Este sufrimiento lo acompañaría por años, una dolencia crónica respiratoria que requiere descanso físico por varias semanas. Igualmente, es clara su vocación y dedicación al Colegio. La carta renuncia es la aseveración del delicado estado de salud y la necesidad de un reposo físico prolongado. Estamos frente a una dolencia crónica respiratoria que mejora con el reposo físico. Es un testimonio de enfermedad y de renuncia al cargo de rector, no es un permiso, por considerar que esta dolencia requiere para su recuperación varias semanas o meses.

Cayetano Heredia regresó a la conducción de la EM el 14 de noviembre de $1842^{(9)}$ y el cuerpo docente de profesores fue nombrado en marzo de 1843 para dar continuidad académica. Asistimos a una discontinuidad académica por razones fiscales. En la hacienda pública, en 1845 , predominaban tres modalidades de finanzas: la hipoteca de los ingresos de aduanas vía bonos, los préstamos forzados y el sistema de abonos que eran pagos adelantados ${ }^{(10)}$. Como será fácil deducir, la economía del EM no era prioridad en regímenes caudillistas militares. Nuestra investigación no encontró documentos sobre actividades académicas en los años 1843 y 1844 .

En septiembre de 1846, el gobierno apoyó la compra de equipos de física que Cayetano Heredia mandó traer desde Europa con economías del colegio. Esta medida está dentro de su ideario no escrito, por lo cual el acto siguiente será contar con un profesor. Para ello consigue que el gobierno, por decreto (diciembre 1846), se autorice al rector contratar un profesor europeo, al cual se costeará el viaje para regentar la cátedra de Química. Este es el punto inicial de un proceso por la construcción de una base científica nacional que apoyaría el desarrollo de la medicina. Un año antes, Cayetano Heredia había informado sobre los problemas económicos del colegio; sin embargo, ahora destina fondos para implementar con docentes y equipos una cátedra importante para la ciencia. Esto nos habla de su capacidad de administrador y organizador. Cambios y avances serán los hitos que veremos en los siguientes años como parte del proyecto implícito de Cayetano Heredia.

Para 1848, luego de varios años de conducción por Cayetano Heredia, la EM ha logrado constituirse en eje de la salud del país, al compendiar en una sola institución ${ }^{(b)}$, asumiendo las facultades de dirigir la formación, licenciar, opinar y ejecutar, por delegación del gobierno, acciones de salud. Igualmente convalida los títulos de médicos extranjeros y faculta su ejercicio. Asimismo actuaba como asesor y en ocasiones ejecutaba medidas de higiene pública. Esta conducción tuvo también espacios donde fue necesario corregir severamente a los estudiantes. En octubre de 1849, Cayetano Heredia informó al ministro del ramo que había procedido a expulsar al estudiante F.P. por grave falta de indisciplina e incumplimiento de sus labores académicas, aplicando el reglamento interno ${ }^{(11)}$.

Once años después del primer documento que nos habla de su enfermedad, volvemos a encontrar otro manuscrito (mayo 1851), que revelará la escasa salud de Heredia. Este testimonio, de su puño y letra, nos habla del progreso de su enfermedad ${ }^{(12)}$ (el subrayado es nuestro):
“... hace seis o siete años que.... principios de invierno me veo atacado por una grave afección del pecho que bajo la forma de asma ha puesto más de una vez en peligro mi existencia. ....... los profesores que se interesan por mi salud y entre ellos el D. Ríos, Solari y Bravo.......me han indicado siempre que el remedio más a propósito a mi padecimiento era variar del temperamento. Pero el vivo deseo que me ha animado por el progreso del Colegio en el que en los últimos años ha sido necesario mucha actividad, no me ha permitido alejarme de él y sólo me he tomado la libertad de ir a bañarme a Chorrillos.... vacaciones. En el presente, en el que del mes de enero me siento más achacoso que nunca, se me han hecho entender.... que debo descansar por dos o tres meses de las fatigas consiguientes... y que mis pulmones deben de ponerse contacto con un aire puro $y$ seco, como el de la sierra para que recobren su antigua energía... y para que al frente de esta casa quede un profesor idóneo y que tenga interés en hacerla prosperar he propuesto... al Dr. Camilo Segura que ha sido vicerrector ... resta sólo Sr. Ministro que si $\mathrm{Ud}$. lo tiene a bien se sirva recabar del $\mathrm{H}$. presidente, la licencia que solicito por tres meses para salir al valle de Jauja...

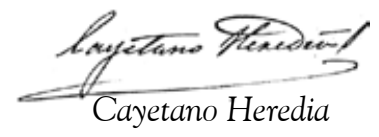

La lectura hace evidente el estado de salud de Cayetano Heredia: once años antes, en 1840, renunció al cargo de rector aduciendo razones de salud; y en este manuscrito confirma que vuelve a repetirse una enfermedad respiratoria que se agudiza periódicamente, con cambios climáticos ('principios de invierno') y estos son desencadenantes de nuevos episodios, a la cual la frasea como... 'asma'... y acudirá en busca de 'aires puros y seco' como el de la ciudad de Jauja. Los profesores que lo atendieron, como D. Ríos era profesor del curso de clínica médica en el hospital San Andrés ${ }^{(13)}$. Asumimos que solo lograría mejoría parcial. En marzo de 1857, E. 
Dornellas, al comentar los lugares adecuados para el reposo de enfermos de tisis, menciona ..."el Perú tiene sus lugares afamados para la residencia de los enfermos atacados de un modo crónico del pecho... los más afamados como Jauja, Tarma ,...." (14). Esta enfermedad respiratoria progresa y lo obligó a guardar reposo, periódicamente, de la cual no es posible concluir en especificaciones por las limitaciones de información. Sin embargo, es posible suponer que esta enfermedad podría haber contribuido a su deceso en junio 1861.

Luego de nueve años, en 1860, su estado de salud siguió deteriorándose. Hemos afirmado de acuerdo a los manuscritos encontrados que desde 1840 se registra un primer deterioro público en su salud; posteriormente, en 1848 y en 1851, y ahora nuevamente en marzo de 1860 vuelve a reiterar

“... mis notorias enfermedades no me permiten asistir, como deseara, a los exámenes anuales que presentan los colegios de esta capital,..." (15), fueron las notas que justifican ante el director de instrucción su inasistencia, a una reunión académica.

Estando por terminar su periodo de tres años como decano, Heredia se dirigió al ministro indicando la conveniencia de renovar su mandato ${ }^{(16)}$. Sin embargo, en abril de 1860, dando cumplimientos a órdenes del gobierno, entregó el cargo al Dr. Miguel De los Ríos. Si bien la comunicación al gobierno por parte de Cayetano Heredia solicita revocar su mandato, icuáles fueron los motivos de su no ratificación en el cargo?, si el reglamento vigente permitía la prórroga por tiempo indefinido. J. Basadre ha sostenido que el reemplazo de Cayetano Heredia fue sorpresivo, decepcionante y humillante para él ${ }^{(17)}$. Heredia había organizado la EM conformando un cuerpo de discípulos con presencia en la sociedad como científicos. Lo cual fue reconocido públicamente por diversos ciudadanos, con notas de agradecimiento que aparecieron, en diferentes fechas, en el diario El Comercio en $1855^{(18-20)}$.
En el aspecto administrativo cumplió con las funciones del cargo con amplitud. Dispuso de parte de su peculio para la formación en el extranjero de algunos de sus discípulos. ¿Qué motivó al gobierno su no ratificación? Autores contemporáneos como U. García (2) han sostenido que el retiro se debió a discrepancias con un sector de médicos, como consecuencia de enfoques diferentes sobre la necesidad del ingreso a la docencia mediante concurso público. Y en un reciente trabajo otro autor sostiene que habría motivaciones políticas ${ }^{(21)}$. Valdizán, en su clásica obra, sostiene "... sólo Ríos podía continuar dignamente la obra emprendida...", mientras que Paz Soldán, sin argumentos, descarta la posibilidad de que la salud fuera un factor de su retiro y anota "... eso no es valedero. Me detengo ante este enigma que no quiero esclarecer..." (1). Era consenso que se había transitado a un mejor momento académico y se proponía una organización académica. Esto habría generado malestares en las esferas allegadas al gobierno, lo cual influiría su no ratificación en el cargo. Es de anotar que su sucesor, Miguel De los Ríos, se mantuvo en el cargo por 20 años. Una reflexión sería que el gobierno tenía conocimiento de su progresiva enfermedad, que cada vez lo limitaba en sus funciones, y prefirió confiar el cargo a una persona vinculada a un sector de profesores, como Miguel de los Ríos, o fue un conjunto de motivaciones políticas y personales de salud física las que explicarían su no ratificación.

Como un gesto de total desprendimiento, Heredia apoyó la formación en Francia de discípulos, para que luego fueran miembros del cuerpo docente ${ }^{(22)}$. Es decir, soñó, pensó, vivió, se consagró y se dedicó en cuerpo y pensamiento a la construcción de una escuela médica peruana siguiendo el mensaje que Hipólito Unanue escribiera en su plan sinóptico. Así termina el período formativo y se consolida la EM en los primeros cincuenta años de su fundación.

Sintiendo el avance de su enfermedad, en su retiro, procedió a realizar testamento el 23 de marzo 1861, disponiendo los fondos económicos para la educación de su hijo, José María Heredia, de 17 años, y la administración de sus rentas ${ }^{(1)}$. Asimismo, continuó preocupado por mejorar los estudios médicos y J. Ulloa anota que "...desde que sintió la proximidad de su fin, el tema de todas sus disposiciones eran los últimos arreglos que tenía proyectados en la Escuela. Había notado hace algún tiempo la falta de un curso de Química analitica,...... Para dictar este curso se necesitaban elementos de no escaso costo. Él se los procuró en medio de sus angustias y este es uno de los legajos que ha dejado al morir,... La biblioteca y el museo necesitaban también algunos objetos: a ellos ha atendido también su magnificencia, dejando para comprarlos otro legajo." (23)

En su retiro, en su casa de Chorrillos, falleció con la penosa enfermedad que lo acompañó en las últimas décadas de su existencia. En sus exequias, entre los numerosos discursos en el camposanto, su más cercano discípulo mencionó "... una enfermedad adquirida, ¿Quién sabe? En los rudos combates de su espíitu lo ha arrebatado a la Escuela y a la ciencia....." (24). Asimismo, en otro momento el alumno Pedro L. Carbajal anota "...pero la enfermedad de que se hallaba atacado el señor Heredia, empezó a hacer rápidos progresos; entonces se presentó una nube en el cielo azul, de nuestra esperanza.... Llegó un día en el cual aquel quedó completamente oscurecido" (25).

Sus discípulos contribuyeron, desde sus publicaciones, a la creación de la salubridad pública en un país que emergía de una crisis política por luchas de bandos militares. Este médico no escribió ningún artículo científico, pero deja una obra singular, la organización de la primera EM del Perú, por lo cual su valor es inmenso al lograr la organización y construcción del aparato académico. Cayetano Heredia organizó las bases de una escuela que posteriormente mostrara aportes interesantes al país. No cabe duda de que el período 1840-1860, con una economía generada por la riqueza del guano y la relativa paz social contribuyeran a las nuevas posiciones de 
la EM, pero solo esto no hubiera sido suficiente; esta es producto del entorno social, pero con hombres que lograron ver mejores tiempos y lo hicieron con proyectos implícitos.

La vida institucional de la primera escuela de medicina, en el periodo inicial de la República, fue crítica, tensa, con graves y profundas dificultades, con momentos que hacían dudar su continuidad. Será la emergencia de un conductor, un organizador, quien logra remontar estos vaivenes, y con un cuerpo de amigos y discípulos logró dar un salto cualitativo. Organiza la primera escuela de medicina de Sudamérica, pasando del modelo miasmático al modelo anatomo clínico, y logra poner a la profesión médica de su segundo plano para un posicionamiento intelectual, científico y sanitario en la sociedad limeña. De igual manera, mediante los trabajos de la escuela contribuyó a la organización de las ciencias médicas, las ciencias naturales y logró importantes intervenciones en la higiene pública y privada.

\section{REFERENCIAS BIBLIOGRÁFICAS}

1. Paz Soldán CE. La vida ejemplar de Heredia. 3ra. Edición. Lima: Universidad Nacional Mayor de San Marcos, 1961

2. Valdizán H. La Facultad de Medicina de Lima 1811 1911, 1ra. Edición. Lima: Imprenta Sanmarti y Co. 1911.

3. Garcia CU. Acta Herediana Cayetano Heredia un simbolo, abril-septiembre 1991. Lima, Perú.

4. Zárate CE. Inicios de la Escuela de Medicina de Lima. Cayetano Heredia el organizador. Asamblea Nacional de Rectores. Lima, 2004.

5. Lastres BJ. Historia de la Medicina Peruana, vol. III. La Medicina en la República, primera edición. Lima: Imprenta Santa María. 1951.

6. Archivo General de la Nación (AGN) L. RJ, ff. 3, 28 marzo 1834

7. Diario El Republicano. Arequipa, 6 de mayo de 1837 tomo $12, \mathrm{~N}^{\circ}{ }^{\circ} 29$

8. AGN RJ 148, ff. 2, 7 de agosto de 1840

9. AGN L. RJ 148, ff. 7. Noviembre 1842.

10. Gutenberg P. Caudillos y Comerciantes. La formación económica del Estado Peruano 1820-1860. Centro de Estudios Regionales Andinos Bartolomé de las Casas, Cusco, Perú. 1997.

11. AGN L .RJ148 ff2, 19 octubre 1849

12. DFML no clasificados ff 2, 14 mayo 1851.

13. Ulloa JC. Escuela de Medicina de Lima, Programa de cursos Gaceta Médica de Lima, 15 mayo 1857.

14. Dornellas E. Hospicio de Tísicos en la isla de Madera. Gaceta Médica de Lima. 30 de marzo 1857.

15. DFML ff. 127-128, 19 marzo de 1860.

16. LNFM ff 129, 21 marzo 1860.
17. Basadre GJ. Historia de la República del Perú, sétima edición, tomo IV. Lima: Editorial Universitaria, 1983: $328 \mathrm{pp}$.

18. El Comercio, 25 julio 1855.

19. El Comercio, 27 julio 1855

20. El Comercio, 8 de noviembre 1855

21. Salaverry O. Personalidades destacadas de la salud pública en el Perú: Cayetano Heredia. Rev Perú Med Exp Salud Publica. 2011;28(3):556-9.

22. LNFM ff 38-39, mayo de 1859.

23. Ulloa JC. El doctor Heredia. Gaceta Médica de Lima. 1861;114:237-40.

24. Ulloa JC. Gaceta médica de Lima. 1861;114:24142.

25. Carbajal PL. Gaceta Médica de Lima. 1861;114:24445.

\section{ABREVIATURAS}

- AGN: Archivo General de la Nación. Lima.

- AFM: Archivos de la Facultad de Medicina de Lima.

- DFML: Documentos en custodia. Facultad de Medicina de Lima.

Artículo recibido el 23 de febrero de 2013 y aceptado para publicación el 6 de mayo de 2013.

El autor declara que no existen conflictos de intereses.

Correspondencia:

Dr. Eduardo Zárate Cárdenas

Correo-e: eduzac@hotmail.com 